EPJ Web of Conferences 75, 05004 (2014)

DOI: 10.1051/epjconf/20147505004

(C) Owned by the authors, published by EDP Sciences, 2014

\title{
Lanthanides in the frame of Molecular Magnetism
}

\author{
D. Gatteschi ${ }^{\mathrm{a}}$
}

Department of Chemistry. INSTM, University of Florence, 50019 Sesto Fiorentino, Italy

\begin{abstract}
Molecular magnetism is producing new types of materials which cover up to date aspects of basic science together with possible applications. This article highlights recent results from the point of view of lanthanides which are now intensively used to produce single molecule magnets, single chain and single ion magnets. After a short introduction reminding the main steps of development of molecular magnetism, the basic properties of lanthanides will be covered highlighting important features which are enhanced by the electronic structure of lanthanides, like spin frustration and chirality, anisotropy and non collinear axes in zero and one dimensional materials. A paragraph of conclusions will discuss what has been done and theperspectives to be expected.
\end{abstract}

\section{A Nano history of molecular magnetism}

Molecular magnetism is a relatively recent section of magnetism which evolved from magnetochemistry. Magnetochemistry is the use of magnetic measurements in order to obtain structural information. An example should explain. Nickel(II) has a configuration $3 \mathrm{~d}^{8}$. A compound with four ligands can have either a tetrahedral or square planar coordination geometry. A ligand field description shows that the five degenerate $d$ orbitals in cubic symmetry remove their degeneracy due to low symmetry effects. The tetrahedral field is weaker than the square planar and the latter forces the electron spins to pair, while the weak tetrahedral keeps two electrons unpaired. A simple magnetic measurement at room temperature decides about the coordination structure. In fact the tetrahedral nickel (II) complexes are paramagnetic and square planar are diamagnetic. A useful technique but nothing more.

The change occurred from the $60 \mathrm{~s}$ of last century when it was realized that molecular chemistry could yield new types of magnetic materials, following the same type of evolution which took place on conductors where the introduction of molecular techniques determined a technological breakthrough.

The goal at the beginning was to design and synthesize high $\mathrm{T}$ organic ferromagnets, but the challenge appeared soon to be too difficult and in order to start from a less hard field the goal was broadened rapidly to molecular ferromagnets i.e. to materials which may contain metal ions together with organic molecules. The results were promising, teaching how to develop strategies to design and synthesize ferro- or ferri-magnetic coupled molecules. The result was the production of low dimensional magnets while 3D materials were more difficult and the number of molecular magnets working at room temperature did not exceed 2 [1-4]. The critical temperatures of pure organic ferromagnets are still in the $30 \mathrm{~K}$ range.

Luckily in those years there was a keen interest among physicists towards low D magnets and the exotic molecular magnets attracted the interest of quite a few. The problem was the different languages of the two communities. Physicists for instance needed one-dimensional antiferro- magnets with $S>1 / 2$ to test the Haldane theory, and the only systems they were aware of classic inorganic systems. The chemists on the other hand had several promising materials but were not aware of the potentialities. Finally the contact of the two communities occurred and they learned that the nickel(II) coordination polymers could be excellent test grounds [5].

Soon there were a number of attempts to communicate between the two communities and I like to remind a NATO ASI held in the Tuscan coast in 1983 where chemists and physicists sat together and talked. In a sense that event 30 years old was the beginning of Molecular Magnetism and the book of proceedings the first text book [6].

The event acted as catalyst and the molecular magnetism became a multidisciplinary field which went much beyond the original expectations. Perhaps

\footnotetext{
${ }^{a}$ Corresponding author: dante.gatteschi@,unifi.it
} 
the most important result was the discovery of single molecule magnets, SMM [7], which was indicated in a special issue of Nature as a milestone in the development of spin in Physics.

SMM was the catchy name invented [8] to define materials whose magnetization below a blocking temperature behaves like that of a magnet, including hysteresis. The behavior is similar to that of a superparamagnet the height of the barrier being of molecular origin. Further SMMs show quantum tunneling effects [9] which generated a great interest because the molecular nature ensures to have available a collection of identical objects, a must to observe quantum effects.

Attempts were done to increase the blocking temperature and rather serendipitously exciting new materials were obtained both containing one dimensional chains, Single Chain Magnets, SCM [1012], and more surprising molecules containing one metal ion, Single Ion Magnets, SIM [13-15].

This was a kind of materials much better suited for molecules and there was soon an explosion of new systems reported and novel properties [16,17].

Lanthanides at the beginning had only marginal interest in MM for several reasons

- $\quad 4 f$ orbitals are internal orbitals and give weak interactions with the surroundings

- Orbital moment is not quenched making the analysis of the magnetic properties difficult

- Anisotropy effects require single crystal investigations

In the last few years however it was found that $\mathrm{Ln}$ containing molecular materials can give rise to unique properties which make the efforts to investigate them well rewarded $[18,19]$.

I wish to briefly highlight some significant results of the last years in the study of molecular magnets containing Ln. The first systems to be covered will be 1D molecules with alternating Gd and radicals which have unique spin frustration conditions and show chiral order, a property of large interest nowadays [20,21]. Similar phenomena are observed in 0D materials in the presence of non-collinear axes.

Before continuing let me explain the spirit I had in writing. The manuscript wants to make available to a broader public the points I made in my presentation. As such I covered only a limited number of topics, and with the bias of a Florentine point of view. I hope the colleagues will not be upset.

\section{Lanthanides and molecular magnetism}

4f orbitals are internal orbitals which interact weakly with the environment. On the other hand the Lns are relatively heavy and the spin orbit coupling effects tend to be more important than Ligand Field effects. This is the scenario for systems containing one $\mathrm{Ln}^{3+}$ ion. The next step is the introduction of exchange interaction, with other Ln or with different magnetic centers like radicals ( $\mathrm{s}$ and $\mathrm{p}$ orbitals involved) or transition metal ions, TM (d orbitals)

In table 1 we show some relevant properties of Ln which will be needed for discussing the properties of the highlighted materials. I show only five ions for the sake of simplicity. The ground state of the ion is characterized by the value of $\mathrm{J}$ shown in Table 1 , and is $2 \mathrm{~J}+1$ times degenerate. The degeneracy is removed by the Ligand field which in tetragonal symmetry yields a series of doublets $\mid \pm M>$, where $M=J, J-1 \ldots$ This gives rise to anisotropy which produces important effects as I will show below.

I will cover first in some detail the exchange interactions between next nearest neighbors, a feature which is known also for transition metal ions, TM, but which has been discovered to have a relevant role in the case of one dimensional materials containing $\mathrm{Ln}$ ions.

Table 1. Basic parameters describing the ground state of

\begin{tabular}{llllll} 
& \multicolumn{5}{c}{${\text { some } \mathrm{Ln}^{\mathrm{a}}}$} \\
Ion & $\mathrm{L}$ & $\mathrm{S}$ & $\mathrm{J}$ & $4 \mathrm{n}$ & $\mathrm{g}$ \\
$\mathrm{Gd}$ & 0 & $7 / 2$ & $7 / 2$ & 7 & 2 \\
$\mathrm{~Tb}$ & 3 & 3 & 6 & 8 & $3 / 2$ \\
$\mathrm{Dy}$ & 5 & $5 / 2$ & $15 / 2$ & 9 & $4 / 3$ \\
$\mathrm{Ho}$ & 6 & 2 & 8 & 10 & $5 / 4$ \\
$\mathrm{Yb}$ & 3 & $1 / 2$ & $7 / 2$ & 13 & $8 / 7$
\end{tabular}

${ }^{\mathrm{a}} \mathrm{L}, \mathrm{S}$ and $\mathrm{J}$ are the orbital, spin, and total quantum numbers. $\mathrm{N}$ is the number of f electrons. $\mathrm{g}$ is the Landè factor

\section{Spin frustration and chirality}

One of the opportunities which have been used to enhance the magnetic properties of $\mathrm{Ln}$ is to let them interact with organic radicals like nitronyl nitroxides, NITR. Very schematically NIT is as sketched below:

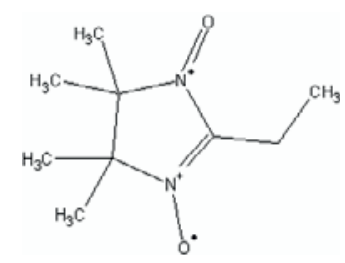

and $\mathrm{R}$ is a substituent group which can vary from a simple $\mathrm{CH} 3$ up essentially to infinite. The unpaired electron is equally delocalized on the two NO groups. The oxygen atoms can bind to two metal ions forming chains of the type

NITR-Ln-NITR-Ln-NITR-Ln-

The exchange interactions between Ln and NITR are of the direct type because the $\mathrm{f}$ orbitals and the $\pi^{*}$ of the radical can have direct overlap $[22,24]$. The nnn interaction can be of the Ln-Ln or of the NITR-NITR type. The simplest case for a qualitative analysis of the 
magnetic properties can be to use $\mathrm{Ln}=\mathrm{Gd}$ because it has no spin orbit coupling in first order and the $S=7 / 2$ is well behaved. Assuming no coupling the system follows the Curie law. Ann ferromagnetic coupling gives a 1D ferromagnetic behavior. Ann antiferromagnetic coupling gives a 1D ferrimagnetic behavior because the two interacting spins are different and do not compensate. In both cases a $\chi \mathrm{T}$ vs $\mathrm{T}$ plot should give a deviation from the $\mathrm{C}_{\mathrm{NITR}}+\mathrm{C}_{\mathrm{Gd}}$ line up while the deviations for different compounds tend down or very slightly up. Relaxing the conditions of including only nn and assuming antiferromagnetic nnn coupling then the system the system becomes spin frustrated as shown in Figure 1. Qualitatively the best compromise is to have two spin up two spin down. However this is based on Ising spins, while both Gd and radical are decisively Heisenberg. Simplified calculations using transfer matrix formalism suggest that the best compromise is to form helices with a pitch which depends on the exchange constants. A picture of the helices is shown in Figure 2.

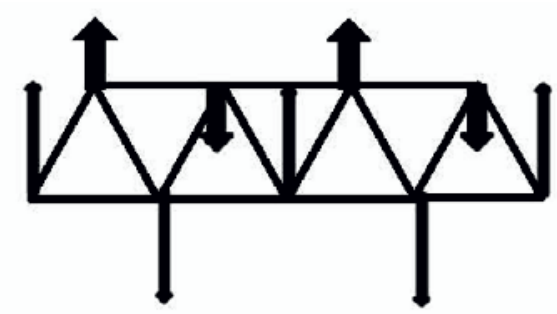

Figure 1. A simplified scheme of the spin arrangement $\mathrm{S}=$ $7 / 2, s=1 / 2$ in the presence of frustration.

Similar chains have been investigated with transition metal ions, TM, but no indication of spin frustration is observed. An indirect proof of the assumption of the antiferromagnetic nnn interaction is the magnetic properties of systems with $\mathrm{Ln}=\mathrm{Y}$ or $\mathrm{Eu}$ which are non magnetic at low $\mathrm{T}$ which clearly show a maximum in the magnetic susceptibility.

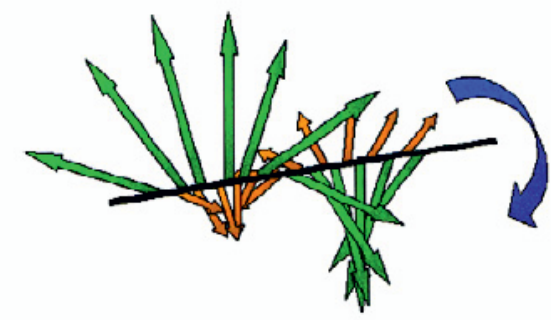

Figure 2. Helical structure of GdNITR chains

A more detailed calculation suggests that the spin correlation is paramagnetic at high temperature while it should give a chirally ordered system at low temperature. In between the two phases there is the possibility of a partially ordered phase which can be discussed starting from the Villain model which he suggested in the 70s but had never been tested due to the lack of suitable materials. The present compounds in fact have been investigated with specific heat and muon spectroscopy. The former evidenced two transitions at and $\mathrm{mK}$ while the latter evidenced one transition at $\mathrm{mK}$. The different behavior is due to the fact that $\mu \mathrm{SR}$ is sensitive to two spin correlation function.

\section{Magnetic Anisotropy and SIM}

Anisotropy can influence the magnetic properties in a dramatic way. A clear example is provided by a Dy NITR compound which has a chain structure analogous to the Gd compound discussed above, DyNITR, which gives rise to magnetic order below $4 \mathrm{~K}$ a quite respectable order temperature for molecular magnets [25]. The origin of this behavior is the relatively strong intra chain interaction which is made more efficient than in the Heisenberg model by the strong Ising (easy axis) magnetic anisotropy. This is enough to enhance the interactions with external chains leading to the ordered state.

The origin of the anisotropy is mainly to be attributed to the single ion contribution [26,27]. An example of the role of single ion anisotropy is provided by a class of molecules which are built up using two square planar macrocycles and one Ln ion. The cyclic molecules have four nitrogen atoms which can bind one metal ion. For TM the metal can fit the N4 cavity while for $\mathrm{Ln}$ the ion is sandwiched between two phthalocyaninate ligands, $\mathrm{pc}^{2-}$ [28]. There is a series of $[\mathrm{NBut}] \mathrm{Ln}(\mathrm{pc}) 2$, where $[\mathrm{NBu}]^{+}$is tetrabutyl ammonium, $\mathrm{Ln}=\mathrm{Gd}-\mathrm{Yb}$. The compounds are generally called double decker and their structure is shown in Figure 3. The choice of metals has been limited to Ln with seven of more electrons in the $4 \mathrm{f}$ orbitals. The unique properties of some of these molecules are that their magnetization becomes blocked at low temperature, in a way similar to that of SCM.

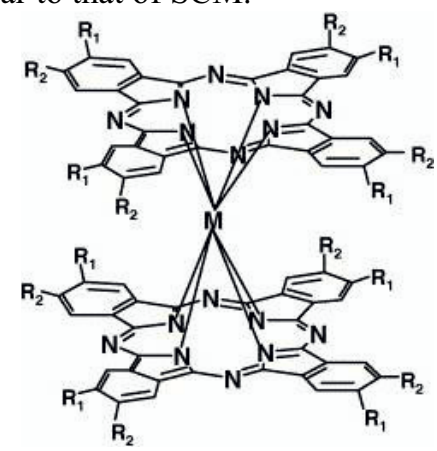

Figure 3. Sketch of the structure of $\operatorname{Ln}(\mathrm{pc}) 2$ -

The origin of the slow relaxation depends on the symmetry break which removes the degeneracy of the 2S+1LJ Russell-Saunders ground state. Let us take as an example $\mathrm{Tb}$, whose relevant parameters are given in Table1. In tetragonal symmetry the ground state breaks down into a number of $\mathrm{M}$ levels, where $\mathrm{M}$ is the projection of J. A series of measurements with different techniques showed that the ground doublet is $\mathrm{M}$. at low $\mathrm{T}$ it will be the only populated one $[29,30]$.

The double decker compounds were not the first SIM, there was an example of similar behavior in 
a Ho doped $\mathrm{YLiF}_{4}$ a compound which was investigated for possible applications in laser diodes, showing that the structural differences do not really mind. The mechanism of relaxation for the inorganic sample was bound to the coupling with the nuclei of Ho. It must be stressed that the interesting magnetic phenomena a micro SQUID operating at $40 \mathrm{mK}$ was needed [31].

Several other series of compounds were found to undergo similar behavior. The best characterized being polyoxo-metallates, POM, which can be considered as the connection between inorganic and molecular materials. They have been used as testing grounds for several different theories, including the open problem of exchange in systems with orbital degeneracy [32].

Another class which is rapidly growing is that which uses cyclooctatetraene, COT, which can also form multiple decker compounds [33].

\section{Anisotropy and non - collinear axes}

Another interesting case is provided by systems in which the local anisotropy axes are not collinear. The simplest case is that of a triangle of three identical spins which are AF coupled as shown in Figure 4. There is a long tradition of investigation of AFM triangles but recently there has been an increase of interest due to the development of Ln based molecular magnets. This is due to the unique properties of lanthanides which have large unquenched orbital moment. The use of techniques which are sensitive to the orientation of local axes is a must if one wants to have a reliable description of the magnetic properties.
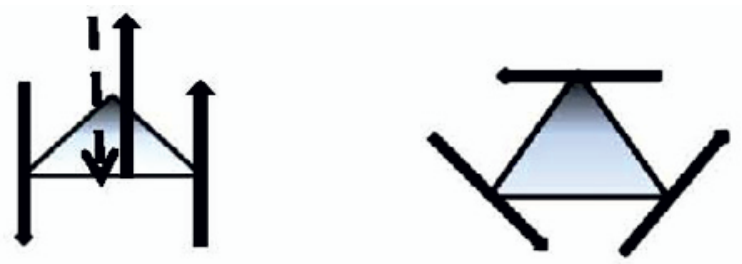

Figure 4. Possible spin arrangements in triangular antiferromagnets

The spins are frustrated and they will have to orient as shown in Figure 4. An important consequence is that there is cancellation of the moment and the magnetic susceptibility goes through a maximum. This behavior is quite unusual for a system with an odd number of unpaired electrons and shows the versatility of $\mathrm{Ln}$.

The experimental data were collected on a sample of a molecule containing a triangle of Dy3 which had been previously prepared. The system was found to undergo quantum relaxation at zero-field and at the field where the excited level crosses the lowest one. This indeed is one of the exciting features of these systems that of producing quantum effects which make them worth investigation for quantum computing.

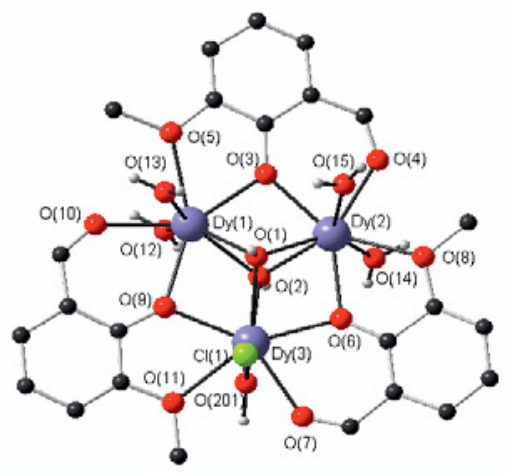

Figure 5. Structure of Dy3

One of the requirements for controlling the quantum tunneling effects is to learn to prepare the material in a given chirality. The strategy for doing that has been efficiently described by Sessoli et al [34,35].

\section{$6 \mathrm{SCM}$}

I have already talked above of one dimensional magnetic materials and want to specialize now to SCM. The originally discovered SCM had chains of alternating cobalt(II) and NITR radicals [10]. The basic properties of SCM were interpreted within the Glauber model [36] a stochastic method to describe the dynamics of one dimensional arrays of objects which are tending to be parallel to each other due to interactions. The model was conceived for ferromagnets but it was applied to completely different objects like liquid crystals, neural networks, phasons social dynamics. However up to the advent of SMM it was not really tested for ferromagnets.

The spin dynamics is developed to give a relaxation of the magnetization of the type

$\tau=\tau_{0} \exp \left(\left(4 \mathrm{~J}+\mathrm{DS}^{2}\right) / \mathrm{KT}\right)$

where $\tau$ is the relaxation time, $\tau 0$ the inverse of the attempt frequency, $\mathrm{J}$ the intra-chain coupling constant, and $\mathrm{D}$ is the parameter of axial second order ligand field.

A system relaxation time increases on decreasing $\mathrm{T}$ and at a given TB becomes blocked. The blockage temperature depends on several parameters and the reliable measurement is done using ac susceptometry where the slowing down of the relaxation is monitored by the appearance of the out of phase component of the measured magnetic susceptibility.

A series of Ln was investigated with hfacligands. $\mathrm{Ln}$ is a metal in the series $\mathrm{Tb}-\mathrm{Yb}$. The $\mathrm{T}$ dependence of the ac magnetic susceptibility clearly shows slow relaxation at low temperature [37]. 


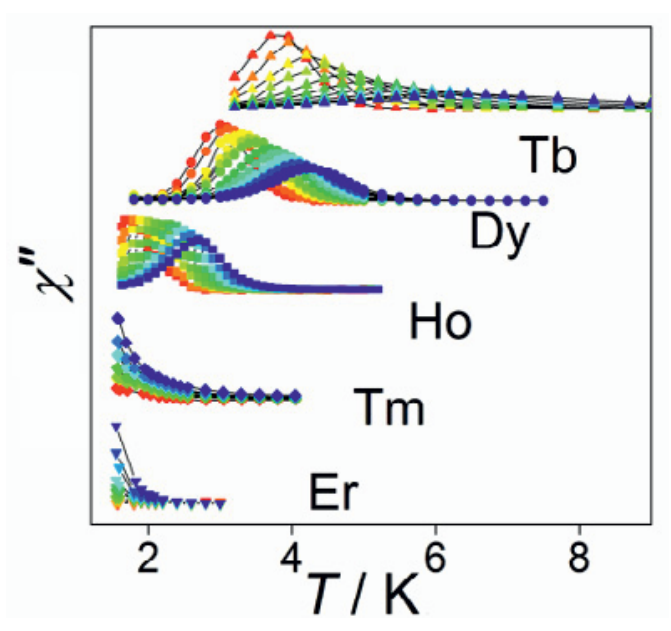

Figure 6.out of phase magnetic susceptibility in the Ln NITR chains

The Dy derivative was also measured in single crystals in order to have a detailed vision of the spin dynamics. The use of angular dependent magnetic measurements was rather frequent at the beginning of the LF description of the electronic structure of TM complexes. The recent availability of sensitive SQUID techniques and the need to obtain detailed knowledge of low symmetry components has determined the development of single crystal magnetic measurements.

The angular dependence of $\chi$ in the range 2$20 \mathrm{~K}$ shows a large anisotropy, with the reversal of the easy and hard axes on varying temperature, with much smaller anisotropy at $20 \mathrm{~K}$. Perhaps the most puzzling feature is the angular dependence of the magnetization in the presence of an applied field of $3 \mathrm{~K}$ as shown in Figure 7.

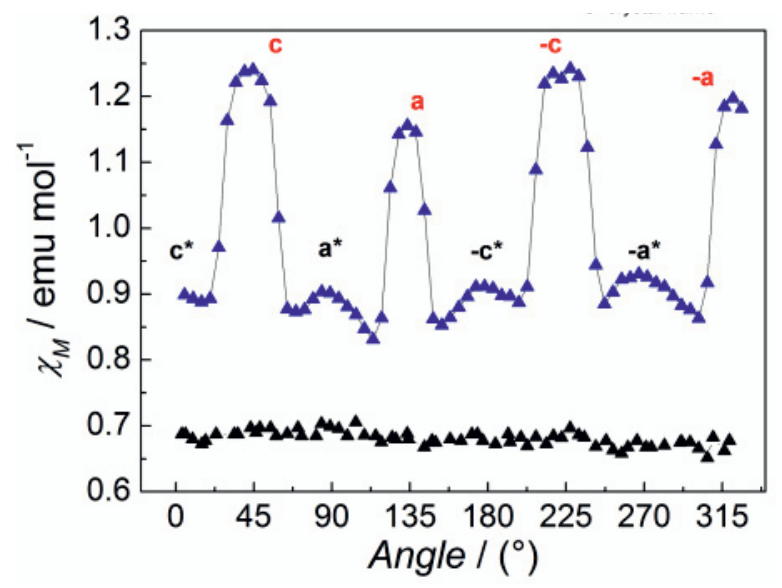

Figure 7

\section{Conclusions}

This short review had the goal to show how MM can provide some interesting new physics. The field is too large to cover it in any detail, so I focused on Lns which have entered the game of molecular magnetism at the beginning of this century.

The current declared goals of molecular magnetism have essentially dropped the dream of room temperature organic ferromagnets. But one never knows.

The dominant dream after the development of SMM was that of single molecule memory storage. After the excitement for SMM and SCM it is SIM to be the most promising and Lns are in one of the top positions. It is the series of multiple decker Ln compounds which has the maximum interest, for their interesting magnetic properties, the possibility of depositing them on suitable surfaces, organizing them and addressing them individually etc. The positive results reported so far have had their origin in the strict multidisciplinary which has been the characteristic of molecular magnetism from the very beginning. And it still is as shown by the numerous papers covering all the aspects with deep insight.

The development of molecular magnetism has been determined by dramatic progress in all the relevant techniques which are needed to make and investigate the new materials. Synthetic chemistry has taken advantage of techniques to design molecules and theoretical approaches are making routine what was wishful thinking. Finally it is amazing how sophisticated measurement techniques are now used.

The most popular keywords at the moment regard the possibility of using $\mathrm{MM}$ in the frame of molecular spintronics. Other possibilities focus on quantum computing, i.e. of using entangled species to define qubits, i.e. novel type of bits based on quantum effects. And quantum effects are under general investigation $[38,39]$.

Finally a less sophisticated field under evaluation is that of using molecular magnets for the development of magnetocaloric effect. This is based on the use of magnetic fields to cool samples. The principle is well known and the new materials may produce important effects at low $\mathrm{T}$.

I mentioned above the NATO ASI which I suggest to be the event which officially started the MM time. The end of the preface concluded: " there was a timely need to bring together the people active in this area to discuss approaches and ideas that can help bring this area of research to maturity" In my opinion there is no doubt that the goal was reached.

\section{References}

1. Weng, D. F.; Wang, Z. M.; Gao, S. Chemical Society Reviews 2011, 40, 3157.

2. Miller, J. S. Chemical Society Reviews 2011, 40, 3266.

3. Train, C.; Gruselle, M.; Verdaguer, M. Chemical Society Reviews 2011, 40, 3297.

4. Magnetism: Molecules to Materials V; Wiley VCH: Weinheim, 2006.

5. Verdaguer, M.; Bleuzen, A.; Marvaud, V.; Vaissermann, J.; Seuleiman, M.; Desplanches, C.; Scuiller, A.; Train, C.; Garde, R.; Gelly, G.; Lomenech, C.; Rosenman, I.; Veillet, P.; Cartier, C.; Villain, F. Coordination Chemistry Reviews 1999, 190-192, 1023. 
6. Stewart, G. A. Hyperfine Interactions 1985, 23, 1.

7. Sessoli, R.; Gatteschi, D.; Caneschi, A.; Novak, M. A. Nature 1993, 365, 141.

8. Aromí, G.; Aubin, S. M. J.; Bolcar, M. A.; Christou, G.; Eppley, H. J.; Folting, K.; Hendrickson, D. N.; Huffman, J. C.; Squire, R. C.; Tsai, H. L.; Wang, S.; Wemple, M. W. Polyhedron 1998, 17, 3005 .

9. Thomas, L.; Lionti, F.; Ballou, R.; Gatteschi, D.; Sessoli, R.; Barbara, B. Nature 1996, 383, 145.

10. Caneschi, A.; Gatteschi, D.; Lalioti, N.; Sangregorio, C.; Sessoli, R.; Venturi, G.; Vindigni, A.; Rettori, A.; Pini, M. G.; Novak, M. A. AngewandteChemie International Edition 2001, 40, 1760.

11. Clérac, R.; Miyasaka, H.; Yamashita, M.; Coulon, C. Journal of the American Chemical Society 2002, 124, 12837.

12. Coulon, C.; Miyasaka, H.; Clérac, R. 2006; Vol. 122, p 163.

13. AlDamen, M. A.; Cardona-Serra, S.; ClementeJuan, J. M.; Coronado, E.; Gaita-Ariño, A.; MartíGastaldo, C.; Luis, F.; Montero, O. InorganicChemistry2009, 48, 3467.

14. Ishikawa, N.; Sugita, M.; Ishikawa, T.; Koshihara, S. Y.; Kaizu, Y. Journal of the American Chemical Society 2003, 125, 8694.

15. Ishikawa, N.; Sugita, M.; Ishikawa, T.; Koshihara, S. Y.; Kaizu, Y. J. Phys. Chem. B 2004, 108, 11265 .

16. Clemente-Juan, J. M.; Coronado, E.; Gaita-Ariño, A. Chemical Society Reviews 2012, 41, 7464.

17. Woodruff, D. N.; Winpenny, R. E. P.; Layfield, R. A. Chemical Reviews 2013, 113, 5110.

18. Bogani, L.; Wernsdorfer, W. Nature Materials 2008, 7, 179.

19. Ren, M.; Pinkowicz, D.; Yoon, M.; Kim, K.; Zheng, L. M.; Breedlove, B. K.; Yamashita, M. Inorganic Chemistry 2013, 52, 8342.

20. Ok, K. M.; Chi, E. O.; Halasyamani, P. S. Chemical Society Reviews 2006, 35, 710.

21. Rogez, G.; Massobrio, C.; Rabu, P.; Drillon, M. Chemical Society Reviews 2011, 40, 1031.

22. Bartolomé, F.; Bartolomé, J.; Benelli, C.; Caneschi, A.; Gatteschi, D.; Paulsen, C.; Pini, M. G.; Rettori, A.; Sessoli, R.; Volokitin, Y. PhysicalReviewLetters1996, 77, 382.

23. Lascialfari, A.; Ullu, R.; Affronte, M.; Cinti, F.; Caneschi, A.; Gatteschi, D.; Rovai, D.; Pini, M. G.; Rettori, A. PhysicalReview B - CondensedMatter and MaterialsPhysics 2003, 67, 2244081.

24. Cinti, F.; Rettori, A.; Pini, M. G.; Mariani, M.; Micotti, E.; Lascialfari, A.; Papinutto, N.; Amato, A.; Caneschi, A.; Gatteschi, D.; Affronte, M. Journal of Magnetism and MagneticMaterials 2010, 322, 1259.

25. Benelli, C.; Caneschi, A.; Gatteschi, D.; Pardi, L.; Rey, P.; Shum, D. P.; Carlin, R. L. InorganicChemistry 1989, 28, 272.

26. Nakano, M.; Oshio, H. Chemical Society Reviews 2011, 40, 3239.
27. Sessoli, R.; Powell, A. K. Coordination Chemistry Reviews 2009, 253, 2328.

28. Ishikawa, N. Polyhedron 2007, 26, 2147.

29. Ishikawa, N.; Sugita, M.; Wernsdorfer, W. AngewandteChemie - International Edition 2005, 44, 2931.

30. Birin, K. P.; Gorbunova, Y. G.; Tsivadze, A. Y. Dalton Transactions 2012, 41, 9672.

31. Giraud, R.; Wernsdorfer, W.; Tkachuk, A. M.; Mailly, D.; Barbara, B. Physical Review Letters 2001, 87, 057203/1.

32. Cardona-Serra, S.; Clemente-Juan, J. M.; Coronado, E.; Gaita-Ariño, A.; Camón, A.; Evangelisti, M.; Luis, F.; Martínez-Pérez, M. J.; Sesé, J. Journal of the American Chemical Society 2012, 134, 14982.

33. Le Roy, J. J.; Jeletic, M.; Gorelsky, S. I.; Korobkov, I.; Ungur, L.; Chibotaru, L. F.; Murugesu, M. Journal of the American Chemical Society 2013, 135,3502 .

34. Tang, J.; Hewitt, I.; Madhu, N. T.; Chastanet, G.; Wernsdorfer, W.; Anson, C. E.; Benelli, C.; Sessoli, R.; Powell, A. K. AngewandteChemie International Edition 2006, 45, 1729.

35. Luzon, J.; Sessoli, R. Dalton Transactions 2012, 41, 13556.

36. Glauber, R. J. Journal of Mathematical Physics 1963, 4, 294.

37. Berlot, K.; Bogani, L.; Caneschi, A.; Gatteschi, D.; Sessoli, R. Journal of the American Chemical Society 2006, 128, 7947.

38. Winpenny, R. E. P. Chemical Society Reviews 1998, 27, 447 .

39. Cornia, A.; Mannini, M.; Sainctavit, P.; Sessoli, R. Chemical Society Reviews 2011, 40, 3076 